medgen $2012 \cdot 24: 73-73$

DOI 10.1007/s11825-012-0317-0

๑) Springer Verlag 2012

\section{NETZWERK 两}

Das Netzwerk Hypophysen- und Nebennierenerkrankungen e.V. ist ein gemeinnütziger Verein von Betroffenen, Angehörigen und Ärzten. Unserer bundesweit tätigen Selbsthilfegruppe haben sich mittlerweile ca. 2400 Patienten angeschlossen. Der Vereinssitz befindet sich in Erlangen. Wir haben zurzeit 28 Regionalgruppen. Sie arbeiten ausschließlich im Rahmen des Netzwerks, unterhalten keinen eigenen Geschäftsbetrieb und erheben keine Beiträge. Inzwischen hat sich sogar eine ausländische Regionalgruppe in Wien/Marienkron gegründet. Wir pflegen auch Kontakte zu ausländischen Patientenselbsthilfegruppen.

Die Gründung des Vereins erfolgte 1994 von damals sechs Patienten und drei Endokrinologen (Fachärzte, die sich auf Hormonstörungen und Stoffwechselerkrankungen spezialisiert haben). Das Netzwerk ist Ansprechpartner und Kontaktadresse für Patienten mit Erkrankungen der Hypophyse (Hirnanhangdrüse) oder der Nebenniere, die wegen der zunächst uncharakteristischen Beschwerden und des langsamen Verlaufes oft sehr spät diagnostiziert und behandelt werden.

Die Patienten leiden unter Morbus Cushing, einem Prolaktinom, der Akromegalie, hormoninaktiven Hypophysenadenomen, Diabetes insipidus, Morbus Addison oder Hypophyseninsuffizienz mit Mangel an Cortisol, Schilddrüsen-, Geschlechts- und Wachstumshormonen und benötigen oft lebenslang Medikamente.

\title{
Netzwerk Hypophysen- und Nebennierenerkrankungen e.V.
}

Bei Patienten mit MEN Typ 1 (multiple endokrine Neoplasie 1) sind sogar mehrere Organe von einer genetisch verursachten Fehlsteuerung betroffen. Dadurch kommt es immer wieder zu Tumorwachstum an hormonproduzierenden Organen. Die Ausprägungen der Tumore sind sehr unterschiedlich und individuell - selbst innerhalb einer Familie - differenziert. Eine lebenslange Kontrolle ist erforderlich, um ein eventuelles Tumorwachstum frühzeitig zu erkennen, da diese Tumore unter Umständen auch entarten können. Die MEN1Mitglieder sind mit einer eigenen Gruppe im Netzwerk vertreten und unterstützen die Arbeit des MEN1-Registers, das am MaxPlanck-Institut, München unter der Leitung von Prof. Ludwig Schaaf geführt wird, ausdrücklich. Ziel dieser Datenbank ist die Schaffung eines Überblicks über betroffene MEN1-Patienten in Deutschland und deren Versorgungssituation. Wissenschaftliche Fragestellungen rund um diese komplexe Erkrankung sollen an möglichst vielen $\mathrm{Pa}$ tienten bearbeitet werden. Die Datenbank soll dazu beitragen, betroffene Patienten und deren Familien noch besser medizinisch betreuen zu können.

\section{Wo wir unsere Aufgaben sehen und wie wir aktiv werden}

Allen Patientengruppen gemeinsam ist der zum Teil oft lange und beschwerliche Weg bis zur Diagnose. Betroffene fühlen sich mit speziellen Fragen häufig allein gelassen.
Deshalb haben wir für Patienten, Angehörige, öffentliche Institutionen und Therapeuten eine Vielzahl von Broschüren erstellt, die sich mit den verschiedenen Hypophysen- und Nebennierenerkrankungen und ihren Therapiemöglichkeiten befassen.

Zweimal im Jahr erscheint unsere Verbandszeitschrift GLANDULA, die sowohl unter Patienten als auch in der Fachwelt hohes Ansehen genießt. Sie wird allen Mitgliedern kostenlos zugeschickt. Da das Netzwerk verstärkt betroffenen Kindern und Jugendlichen helfen will, wird seit 2008 außerdem die Beilage "Glandulinchen“ publiziert, die sich speziell mit diesem Themenbereich befasst. Sie erscheint einmal jährlich.

Für einige unserer Erkrankungsbilder haben wir Patienten als direkte Ansprechpartner. Der Vorstand ist mit Betroffenen besetzt und wird von einem wissenschaftlichen Beirat von Fachärzten unterstützt.

Außerdem veranstalten wir jährlich an wechselnden Orten den überregionalen Hypophysen- und Nebennierentag, der regelmäßig große Resonanz findet. Neben einem breiten Angebot an Fachvorträgen besteht hier auch ausgiebig Gelegenheit zum Erfahrungsaustausch. Außerdem werden regionale Hypophysen- und Nebennierentage und eine Vielzahl regelmäßiger Regionaltreffen organisiert. Die Regionalgruppenleiter treffen sich als wichtige Multiplikatoren einmal im Jahr zum Erfahrungsaustausch und zur Erörterung organisatorischer Fragen.

Des Weiteren fördert das Netzwerk Seminare und Weiter- bildungsmaßnahmen für Betroffene und Ärzte und unterstützt die medizinische Forschung.

Mitglieder und Betroffene können uns an vier Tagen in der Woche telefonisch erreichen. Außerdem ermöglichen wir im Internet (www.glandula-online.de) mit einer breiten Auswahl an Foren einen vielfältigen Austausch. Auf unserer umfangreichen Webseite werden unter anderem aktuelle Neuigkeiten veröffentlicht und unsere Broschüren zum kostenlosen Download angeboten.

Geschäftsstelle

Netzwerk Hypophysen- und Nebennierenerkrankungen e.V. Waldstr. 53

90763 Fürth

Telefon 0911-9792009-0

Telefax 0911-9792009-79

Email: netzwerk@glandula-online.de 